\title{
Pattern of Antimalarial Drug Use in Malaysia
}

\author{
N. M. ARIFFIN, F. ISLAHUDIN*, M. MAKMOR-BAKRY, E. KUMOLOSASI, R. N. MUDIN, U. K. SHAMSUDIN, M. H. A. \\ HAMID $^{1}$, W. M. KEONG ${ }^{1}$, A. H. S. M. HAQ ${ }^{2}$ AND S. F. ABU ${ }^{2}$ \\ Faculty of Pharmacy, Universiti Kebangsaan Malaysia, Jalan Raja Muda Abdul Aziz, 50300, Kuala Lumpur, \\ ${ }^{1}$ Vector Borne Disease Sector, Disease Control Division, Ministry of Health, Malaysia, Level 4, Block E10, \\ Complex E, Central Federal Government Administration, 62590, Putrajaya, 2Pharmaceutical Services Division, \\ Lot 36, Jalan Universiti, 46200, Petaling Jaya, Malaysia
}

\section{Ariffin, et al.: Antimalarial Drugs in Malaysia}

This work aims to identify the pattern of antimalarial use in Malaysia as a baseline data on common drugs used in the local setting. Data on malaria prevalence and drug use pattern over the past 10 years was collected retrospectively from Vector Borne Disease Sector and Pharmaceutical Services Division, Ministry of Health. The defined daily dose/inhabitant/years are used to describe the antimalarial utilization pattern. We observed a decline of $\mathbf{4 2 \%}$ malaria cases within a span of 10 years despite a steady increase in the number of Plasmodium knowlesi cases noted in Malaysia. Infections caused by the $P$. falciparum and $P$. vivax species demonstrated a reduction in cases within the span of 10 years. Between 2005 and 2013 both chloroquine and primaquine were noted to be the most common antimalarial drugs used, followed by pyrimethamine-combination and quinine. Artemisinin in particularly artesunate and mefloquine use were found to be limited during the study period. Despite being one of the oldest drugs, the quinolines were found to be the most common antimalarials used. The use of primaquine is highlighted due to the gradual increase in the use of the drug in recent years. Indeed, the general decrease in antimalarial use is attributed to the distinct reduction in malaria cases in the country.

Key words: Malaria, drug use pattern, antimalarial drugs, defined daily dose

Global burden in tackling malaria infection in order to avoid disease progression is a challenging issue. The impact of malaria is staggering, despite the various interventions and strategies executed ${ }^{[1]}$. Due to its dynamic nature that involves spread of disease through travels and migratory movement, malaria has been found to be difficult to eradicate completely ${ }^{[2,3]}$. It affects a large number of countries especially in Africa and Asia and can cause death in individuals who are not immunized through natural immunity. In view of the high mortality rate, especially in children, a prompt approach in tackling the disease, monitoring drug use and a new approach in treatment is crucial.

The use of antimalarials for treating and eradicating

*Address for correspondence E-mail: faridaislahudin@ukm.edu.my

May - June 2016 malaria infections in Malaysia has been established nationwide $^{[4]}$ and is in accordance with latest WHO guidelines $^{[5]}$. However, it is important to note that there has been a slow decline in introduction of new antimalarial drugs over the past few years with treatment dependent on only a handful of drugs ${ }^{[6]}$. Furthermore, with recent trends in resistance of Plasmodium sp. towards older antimalarials such as chloroquine and

This is an open access article distributed under terms of the Creative Commons Attribution-NonCommercial-ShareAlike 3.0 License, which allows other the remix, tweak, and build up to the non-commercially, as long as the author is credited and the new creations are licensed under the identical terms. 
quinine, as well as newer artemisinins ${ }^{[7]}$, continuous monitoring of is required to ensure that drug efficacy is preserved. Close monitoring of malaria treatment is also required to ensure that drug safety and optimal efficacy.

Malaysia has managed to ensure that malaria infection is controlled. The decline in infections has been steady over the years ${ }^{[8]}$. Although eradication is far from near, Malaysia has prioritized malaria control with a clear focus in malaria elimination as endorsed through the specific National Strategic Plan for Elimination of Malaria (2011-2020). Although urban areas have shown a decline in malaria infection, many rural areas are still at risk of infection. Therefore, this work aims to report the trends in malaria infection and antimalarial use in Malaysia to identify the pattern of infection and drug use over the past few years.

A cross-sectional, retrospective study was conducted from 2005-2014. The study was registered in the national medical research registration. Ethical approval was obtained from the National Medical Research Ethics Committee (ID: NMRR-14-1308-23222).

Information on malaria prevalence was extracted from the electronic database of the Vector Borne Disease Sector, Ministry of Health. Data included were demographic information such as gender, ethnic, age, prevalence of malaria infection, species of malaria parasite causing human malaria infection and type of malaria transmission.

Data on antimalarial drug were obtained from the National Medicines Use Survey for year 2005-2013. The survey was conducted by Pharmaceutical Services Division and jointly sponsored with the Clinical Research Centre, Ministry of Health. Data sources and surveys presented in this report are primarily derived from medicine procurement and prescriptions in public health institutions. The process of gathering data medicines consumption and sales involves multiple surveys at the various levels of the medicines supply and distribution chain in the country (Division and Centre $)^{[10]}$.

The measurement for antimalarial drug utilization trend is expressed in defined daily dose (DDD) ${ }^{[9]}$. The DDDs/inhabitant/years is used to describe the antimalarial utilization pattern as it is normally used for items prescribed for short durations ${ }^{[10]}$. This method gives an estimation of the average number of days for which inhabitants are treated annually. For example, an estimate of five DDDs per inhabitant per year indicates that the utilization is equivalent to the treatment of every inhabitant with a 5-day course during a certain year.

Malaria control programs in Malaysia were initiated as far back as the 1960s. The latest initiative is the focus on elimination of malaria by the year 2020, through the National Malaria Elimination Strategic Plan 2011$2020^{[11-13]}$. In Malaysia, results demonstrated that a total of 55173 confirmed malaria cases were notified in the 10 year period between 2005 and 2014. In general, there was a $42 \%$ steady decline of malaria cases from 2005 to 2014. Similarly, mortality trend was also observed to decline steadily from 33 cases in 2005 to only 9 cases in 2014. Evidently, the decline in malaria infection in Malaysia is attributed to the effective implementation strategies through the national malaria surveillance system $^{[8]}$. The current malaria control program performs a high degree of vigilance and robust surveillance to ensure vector control especially in receptive areas. This includes rapid testing, confirmatory testing of all suspected cases, mandatory reporting of malaria cases, integrated vector management and trained primary healthcare workers in malaria management ${ }^{[14,15]}$. However, despite the significant reduction in malaria cases within the past 10 years, elimination of malaria may still remain a challenge.

The demographic characteristic of malaria cases was predominantly male $(n=42,388 ; 77 \%)$ compared to female $(n=12,785 ; 23 \%)$. Predominantly infecting young males, the disease has a major impact on this particular population especially as those that are affected tend to be among the agricultural and outdoor workers $^{[14]}$. A small percentage of women that were infected with malaria were among those that were pregnant $(n=269,1.9 \%)$. Those that were infected with malaria were found to be between the ages of $<1$ y to $>55 \mathrm{y}$ old with the majority being between the ages of $15-34$ y old ( $n=26,127 ; 47.4 \%)$. A large number of malaria cases were noted to be among locals $(n=36,237$, $65.7 \%$ ) with the remaining cases of malaria from foreigners $(n=18,936 ; 32.3 \%)$. Indeed, data also shows that the migration of workers from endemic countries also contribute to a significant number of malaria cases in Malaysia. Therefore, identifying alternative ways in optimizing management of malaria may provide better control of the infection.

As observed, the current main infections over the past 10 years are caused by $P$. vivax $(\mathrm{n}=24,952 ; 45.2 \%)$ and P. falciparum (n=14,465; 26.2\%, fig. 1). P. falciparum 
species was predominantly identified in East Malaysia such as Sabah and Sarawak. P. falciparum is able to cause a more serious and life-threatening infection if treatment is delayed. The majority of $P$. vivax was mainly found in Peninsular Malaysia, most notably in Selangor. In addition, the total of relapse cases $(\mathrm{n}=107$ from 2011-2014) were mainly found in $P$. vivax infections compared to other malaria species. Although $P$. vivax is less severe, complications caused by the species have been reported ${ }^{[16]}$. Infections from $P$. knowlesi and P. malariae were 16\% $(\mathrm{n}=8993)$ and $9 \%$ $(\mathrm{n}=5091)$, respectively, whereas $P$. ovale $(\mathrm{n}=43,0.07 \%)$ caused the least number of infections in Malaysia. Within the study duration, there was also a total of $1629(2.9 \%)$ mixed infections that were identified. Nonetheless, recent epidemiology of malaria parasite transmission has changed slightly within the past few years with the emergence of $P$. knowlesi ${ }^{[8,17]}$. Despite a steady decline in $P$. falciparum and $P$. vivax cases, the trend in $P$. knowlesi has been increasing steadily and surpasses other malaria parasites since 2012 (fig. 1). This is especially true for Sabah, in which approximately $60 \%$ of cases are caused by $P$. knowles $i$ in $2014{ }^{[8]}$. Interestingly, current data suggests that in Peninsular Malaysia, the main malaria parasites involved are $P$. vivax and $P$. knowlesi, which account for more than half of malaria infections ${ }^{[8]}$.

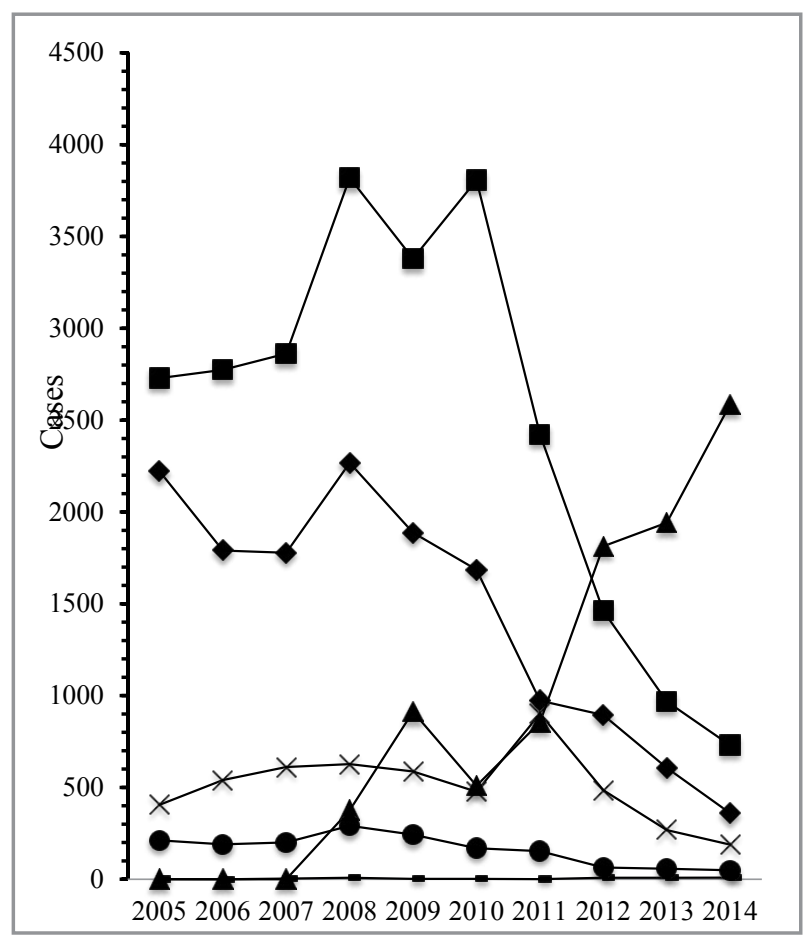

Fig. 1: Distribution of malaria parasite causing human infection 2005-2014.

Y-axis: number of cases, X-axis: year 2005-2014. Symbols in the figure are represented by ( $\$$ ) . falciparum, (ロ) P. vivax, (×) $P$.

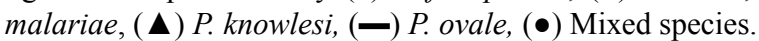

Despite being some of the oldest drugs, the quinolines were found to be the most common antimalarials used, which reflects the management of malaria infections observed in the current work. However, the overall data suggests that the use of antimalarial is reducing from 2005 to 2013 (fig. 2). Indeed, the general decrease in antimalarial use is attributed to the distinct reduction in malaria cases in the country. Quinine and chloroquine were used as first line management of $P$. falciparum and $P$. vivax prior to 2007 . However, this has given way to the newer artemisinins with quinine being used as second-line treatment ${ }^{[4,18]}$. Between 2005 and 2013 both chloroquine and primaquine were noted to be the most common antimalarial drugs used, followed by pyrimethamine combination and quinine (fig. 3). Interestingly, despite the reduction in overall antimalarial use, primaquine remains the highest drug used from 2006 (0.0533 DDD/population/year) to 2013 (0.0036 DDD/population/year). The high use of primaquine in Malaysia reflects the additional use of primaquine for $P$. falciparum for gametocidal effect. Thus, despite a reduction in the older quinine and chloroquine, the use of primaquine was found to increase significantly in recent years. Artemisinin drug, particularly artesunate and mefloquine use were found to be limited during the study period.

Primaquine is the only drug used for the radical cure of $P$. vivax and $P$. ovale ${ }^{[19]}$. It holds a unique place in malarial treatment due to the mechanism of primaquine that affects hypnozoite stages of the parasite, making it valuable in preventing relapse caused by $P$. vivax and $P$. oval $e^{[19]}$. On the other hand, it has gametocidal effects that require only a single dose to be used in

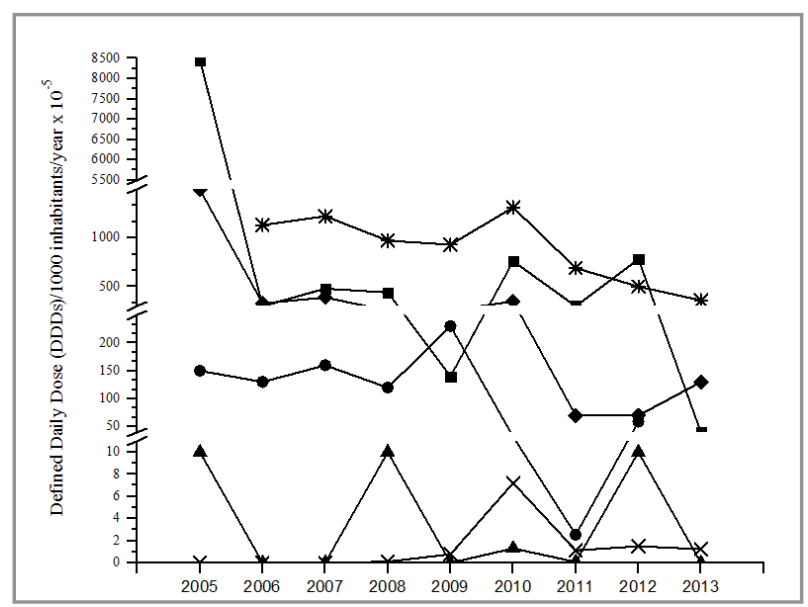

Fig. 2: Antimalarial use from 2005 to 2013.

Y-axis: Defined Daily Dose (DDD)s/1000 inhabitants/year $\times 10^{-}$ 5, X-axis: year 2005-2013. Antimalarials covered were (ם) chloroquine, $\left(^{*}\right)$ primaquine, $(\boldsymbol{\nabla})$ pyrimethamine combination, $(\bullet)$ quinine, $(\boldsymbol{\Delta})$ mefloquine and $(\times)$ artemisinin derivatives. 


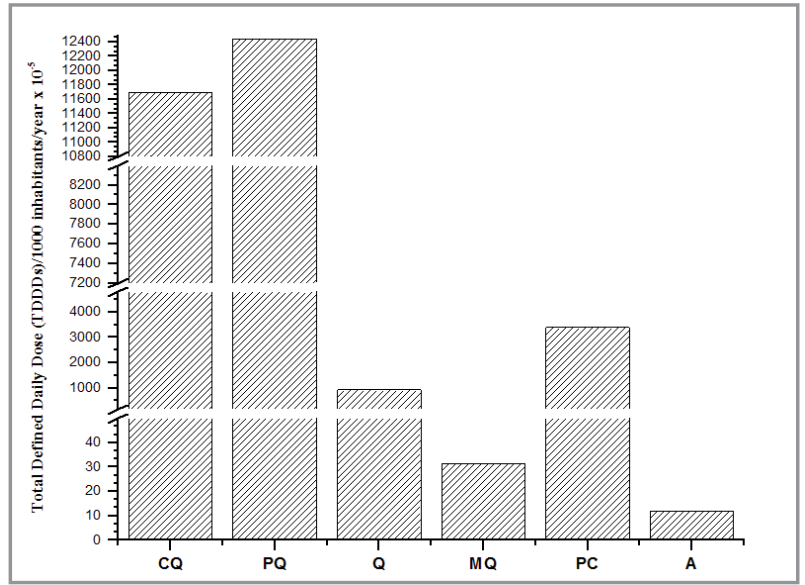

Fig. 3: Total defined daily dose of antimalarial by drugs between 2005 and 2013.

Y-axis: Defined Daily Dose (DDD)s/1000 inhabitants/year $\times 10^{-5}$, $\mathrm{X}$-axis: antimalarial drugs represented by $\mathrm{CQ}$ : chloroquine, $\mathrm{PQ}$ : primaquine, QN: quinine, MQ: mefloquine, $\mathrm{PC}$ : pyrimethamine combination, $\mathrm{A}$ : artemisinin derivatives

management of $P$. falciparum. The single dose is used often with artemisinin. The effectiveness of the drug makes it one of the most valuable antimalarials to date. The importance of pharmacogenetics in primaquine use is evident with the discovery of glucose-6-phosphate dehydrogenase (G6PD) deficiency ${ }^{[20]}$. In view of this doses of primaquine are dependent on the G6PD status of the patients and ranges from $15 \mathrm{mg}$ to $45 \mathrm{mg}$ on a daily or weekly basis ${ }^{[21,22]}$. This reduces the risk of severe adverse reactions occurring in patients. Similarly, genetic work has demonstrated therapeutic effects are dependent on other genetic polymorphisms ${ }^{[23]}$, which could potentially lead to ineffectiveness and the risk of relapse. Furthermore, work has shown that the potential use of primaquine in $P$. Knowlesi ${ }^{[24]}$ could possibly increase the use of primaquine in the future.

Therefore, the aim of the study to identify the pattern of antimalarial use was successfully achieved. The use of primaquine is highlighted due to the gradual increase in the use of the drug in recent years. Primaquine is currently indicated for management of $P$. falciparum, $P$. vivax and $P$. ovale with potential use in $P$. knowlesi. In an attempt to ensure effective use of the drug and preventing treatment failure, identifying ways to optimise the use of primaquine should be performed in the future.

\section{Acknowledgments:}

We acknowledge the support of the Vector Borne Disease Sector Disease Control Division and Pharmaceutical Services Division, Malaysia Ministry of Health in the preparation of this document. We would also like to thank the Director General of Health, Malaysia for his permission to publish this article.

\section{Financial support and sponsorship:}

Nil.

\section{Conflicts of interest:}

There are no conflicts of interest.

\section{REFERENCES}

1. Lozano R, Naghavi M, Foreman K, Lim S, Shibuya K, Aboyans V, et al. Global and regional mortality from 235 causes of death for 20 age groups in 1990 and 2010: A systematic analysis for the Global Burden of Disease Study 2010. Lancet 2012;380:2095-128.

2. Martens P, Hall L. Malaria on the move: human population movement and malaria transmission. Emerg Infect Dis 2000;6:103-9.

3. Bhutta ZA, Sommerfeld J, Lassi ZS, Salam RA, Das JK. Global burden, distribution, and interventions for infectious diseases of poverty. Infect Dis Poverty 2014;3:21.

4. Vector Borne Disease Sector Disease Control Division, Ministry of Health. Management Guideline of Malaria in Malaysia. 2014.

5. Guidelines for the Treatment of Malaria. 3rd edition. Geneva: World Health Organization; 2015. Available from: http:// www.ncbi.nlm.nih.gov/books/NBK294440/

6. Schlitzer M. Antimalarial drugs - what is in use and what is in the pipeline. Arch Pharm (Weinheim) 2008;341:149-63.

7. Ashley EA, Dhorda M, Fairhurst RM, Amaratunga C, Lim P, Suon S, et al. Spread of Artemisinin Resistance in Plasmodium falciparum Malaria. N Engl J Med 2014;371:411-23.

8. Vector Borne Disease Sector Disease Control Division, Ministry of Health. Malaria Situation in Malaysia, 2014.

9. WHO Collaborating Centre for Drug Statistics Methodology, Guidelines for ATC classification and DDD assignment 2013. Oslo, 2012.

10. Pharmaceutical Services Division and Clinical Research Centre, Ministry of Health. Malaysian Statistics on Medicines 2009 and 2010.

11. Rundi C. Malaria Elimination in Malaysia. Third annual meeting of the Asia Pacific Malaria Elimination Network (APMEN); Kota Kinabalu, Sabah, Malaysia; 2011.

12. APMEN. Asia Pacific Malaria Elimination Network. Available from: www.apmen.org

13. Hsiang MS, Abeyasinghe R, Whittaker M, Feachem RG. Malaria elimination in Asia-Pacific: an under-told story. Lancet 2010;375:1586-7

14. Rahman H. Updates on Malaria Control Activities in Malaysia. First annual meeting of the Asia Pacific Malaria Elimination Network (APMEN); Brisbane, Australia; 2009.

15. Asian Collaborative Training Network for Malaria. MalaysiaCountry Profile: 2008. Available from: www.actmalaria.net

16. Barber BE, William T, Grigg MJ, Menon J, Auburn S, Marfurt J, et al. A prospective comparative study of knowlesi, falciparum and vivax malaria in Sabah, Malaysia: high proportion with severe disease from Plasmodium knowlesi 
and $P$. vivax but no mortality with early referral and artesunate therapy. Clin Infect Dis 2013;56:383-97.

17. William T, Rahman HA, Jelip J, Ibrahim MY, Menon J, Grigg MJ, et al. Increasing incidence of Plasmodium knowlesi malaria following control of $\mathrm{P}$. falciparum and $P$. vivax malaria in Sabah, Malaysia. PLoS Negl Trop Dis 2013;7:e2026.

18. Pharmaceutical Sevices Division, Malaysia Ministry of Health. National Antibiotic Guideline 2014. 2nd Ed, 2015.

19. Hill DR. Baird JK, Parise ME, Lewis LS, Ryan E, Magill AJ. Primaquine: report from CDC expert meeting on malaria chemoprophylaxis I. Am J Trop Med Hyg 2006;75:402-15.

20. Howes RE, Battle KE, Satyagraha AW, Baird JK, Hay SI. G6PD deficiency: global distribution, genetic variants and primaquine therapy. Adv Parasitol 2013;81:133-201.

21. Buchachart K, Krudsood S, Singhasivanon P, Treeprasertsuk
S, Phophak N, Srivilairit S, et al. Effect of primaquine standard dose (15 mg/day for 14 days) in the treatment of vivax malaria patients in Thailand. Southeast Asian J Trop Med Public Heal 2001;32:720-6.

22. White NJ, Qiao LG, Qi G, Luzzatto L. Rationale for recommending a lower dose of primaquine as a Plasmodium falciparum gametocytocide in populations where G6PD deficiency is common. Malar J 2012;11:418.

23. Kerb R, Fux R, Morike K, Kremsner PG, Gil JP, Gleiter $\mathrm{CH}$, et al. Pharmacogenetics of antimalarial drugs: effect on metabolism and transport. Lancet Infect Dis 2009;9:760-74.

24. Daneshvar C, Davis TM, Cox-Singh J, Rafa'ee MZ, Zakaria SK, Divis PC, et al. Clinical and parasitological response to oral chloroquine and primaquine in uncomplicated human Plasmodium knowlesi infections. Malaria J 2010;9:238. 